\title{
Selective Reduction in Neural Responses to High Calorie Foods Following Gastric Bypass Surgery
}

\author{
Christopher N. Ochner, PhD ${ }^{\star}, \dagger$, Yolande Kwok, MHSA*, Eva Conceição, MS ${ }^{*}$, Spiro P.

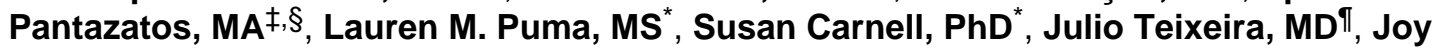 \\ Hirsch, PhD $\$$, and Allan Geliebter, $\mathbf{P h D}^{\star}, \dagger$ \\ *New York Obesity Research Center, St. Luke's Roosevelt Hospital, New York \\ † Department of Psychiatry, Columbia University College of Physicians and Surgeons, New York \\ ‡ Department of Physiology and Cellular Biophysics, Columbia University Medical Center, New \\ York \\ $\S$ Program for Imaging and Cognitive Sciences, fMRI Research Center, Columbia University \\ Medical Center, New York \\ I Center for Bariatric Surgery and Metabolic Disease, Department of Minimally Invasive Surgery, \\ St. Luke's Roosevelt Hospital, Columbia University College of Physicians and Surgeons, New \\ York
}

\begin{abstract}
Objective-To investigate changes in neural activation and desire to eat in response to appetitive cues from pre- to postbariatric surgery for obesity.

Background-Roux-en-Y gastric bypass (RYGB) is the most common bariatric procedure. However, the mechanisms of action in RYGB are not well understood. A significant proportion of the resulting reduction in caloric intake is unaccounted for by the restrictive and malabsorptive mechanisms and is thought to be mediated by neuroendocrine function. Numerous investigations of postsurgical changes in gut peptides have resulted; however, changes in neural activation after RYGB surgery have not been previously investigated.
\end{abstract}

Methods-Functional magnetic resonance imaging and verbal rating scales were used to assess brain activation and desire to eat in response to high-and low-calorie food cues in 10 female patients 1-month pre- and post-RYGB surgery.

Results-Postsurgical reductions in brain activation were found in key areas within the mesolimbic reward pathway, which were significantly more pronounced in response to food cues that were high (vs. low) in caloric density. These changes mirrored concurrent postsurgical reductions in desire to eat, which were also greater in response to food cues that were high versus low in caloric density $(P=0.007)$.

Conclusions-Findings support the contention that RYGB surgery leads to substantial changes in neural responses to food cues encountered in the environment, provide a potential mechanism for the selective reduction in preferences for high-calorie foods, and suggest partial neural mediation of changes in caloric intake seen after RYGB surgery.

Copyright $\odot 2011$ by Lippincott Williams \& Wilkins

Reprints: C. Ochner, NY Obesity Research Center, St. Luke's Roosevelt Hospital Center, 1111 Amsterdam Avenue, Babcock 1020, New York, NY 10025.co2193@columbia.edu.

Supplemental digital content is available for the article. Direct URL citations appearing in this printed text are provided in the HTML and PDF version of this article on the journal's web file (www.annalsofsurgery.com). 
Obesity has reached pandemic proportions, ${ }^{1}$ with bariatric surgery representing the only currently available treatment demonstrating long-term effectiveness. ${ }^{2}$ Roux-en-Y gastric bypass (RYGB) remains the most common and effective bariatric procedure used today, accounting for approximately $65 \%$ of the procedures performed worldwide. ${ }^{3}$ However, the mechanisms that lead to reduced caloric intake and weight loss after RYGB are poorly understood. ${ }^{4-7}$ Although the restricted $(15-50 \mathrm{~mL})$ postsurgical pouch limits ingestive capacity $^{2,5}$ and the bypassing of the upper portion of the small intestine may prevent a percentage of ingested calories from being absorbed into the body, $2,5,8$ these mechanisms do not fully account for the associated postsurgical weight loss. ${ }^{4-7,9-14}$ The neurohormonal system has been implicated in accounting for at least some proportion of the postsurgical weight loss not explained by these changes in gut physiology. $., 5,7,13,14$ Numerous investigations of appetite-related gut peptides have resulted; ${ }^{4,7,13,14}$ however, there have been only 2 preliminary $\left(\mathrm{N}=5\right.$ in each) positron emission tomography studies ${ }^{15,16}$ of D2 receptor availability in the striatum before and after gastric bypass surgery, and no published investigations of changes in neural activity after RYGB using functional magnetic resonance imaging (fMRI). Further, no studies have specifically examined whole brain responses to food cues postsurgery, which may give a more comprehensive picture of neural changes contributing to postsurgical decreases in appetite and weight.

The homeostatic regulation of energy balance (ie, food intake) is largely mediated by hypothalamic activity. ${ }^{17}$ Alternately, the perceived reward or "hedonic" value of external stimuli, including food, ${ }^{18}$ is mainly processed within the mesolimbic reward pathway. This system operates primarily through dopaminergic signaling 17 to form judgments and initiate behavioral responses based on the perceived reward value of food cues encountered in the environment. ${ }^{19}$ A number of areas within the mesolimbic pathway contribute to reward value appraisals of food cues, including the ventral tegmental area (VTA), amygdala, hippocampus, and ventral striatum, ${ }^{17,18}$ all falling under the executive control of the prefrontal cortex (PFC). ${ }^{18-20}$ The PFC integrates reward-related information from other corticolimbic areas and sensory information (primarily) from the thalamus, ${ }^{17,20}$ and is responsible for initiating behavioral responses (ie, whether or not to consume food) based on hedonic appraisals of food cues. ${ }^{17,18,20}$ Some increases in activation within the mesolimbic pathway have been found after caloric restriction, ${ }^{21}$ which may explain the heightened desire to eat reported during weight loss maintenance. ${ }^{21,22}$ However, such increases in desire to eat are not seen after RYGB,,${ }^{9,10}$ even upon exposure to highly palatable food cues. ${ }^{9}$

Highly palatable and high energy density (ED; kcal/g) food cues are ubiquitous in most industrialized nations. ${ }^{18}$ Exposure to such cues has been shown to activate the mesolimbic reward pathway ${ }^{17,18}$ and predict food intake. ${ }^{23}$ Given consistent postsurgical reductions in the desire for and intake of food, ${ }^{9,10}$ it was hypothesized that participants undergoing RYGB would show postsurgical reductions in mesolimbic reward pathway activation in response to food cues. An additional phenomenon associated with bypass procedures is the selective reduction in the preference for highly palatable high-ED foods. ${ }^{9-12}$ After RYGB surgery, presurgical preferences for foods high (vs. low) in fat and calories are reduced or eliminated. ${ }^{9-12}$ Thus, it was anticipated that postsurgical reductions in mesolimbic activity would be greater in response to high-ED relative to low-ED food cues. Finally, a related but unexplored concept is that of appetitive responsivity or the desire to eat (anything) after exposure to food cues. ${ }^{24}$ Given selective postsurgical reductions in the drive to consume high-ED foods, ${ }^{9-12}$ we predicted a similar selective postsurgical reduction in desire to eat after exposure to high- relative to low-ED food cues. 


\section{METHODS}

\section{Participants}

Twelve female RYGB surgery candidates were recruited from the Center for Weight Loss Surgery at a large university-affiliated hospital in New York City. Scans from 2 participants were discarded due to missing or corrupt data, leaving 10 completers. Participants ranged in presurgical body mass index (BMI) from 40 to $54 \mathrm{~kg} / \mathrm{m}^{2}$ [mean $=45 \pm 5$ (SD)] and age from 20 to 47 years (mean $=35 \pm 9$ ), were weight-stable, right-handed, nonsmoking, premenopausal, free of any major psychological or physical disorders (including diabetes) and were not taking any medication that may have affected body weight. Fifty percent of the sample was Hispanic, 30\% African American and 20\% white. Institutional review board approval for this study was granted by Columbia University and St. Luke's Roosevelt Hospital. All participants provided informed consent and met the criteria proposed by the National Institutes of Health Consensus Panel. ${ }^{25}$

\section{Design and Procedure}

A within subjects design was used with assessments at 1-month pre- and 1-month postRYGB surgery. After an overnight (12 h) fast, participants reported to the fMRI Research Center at the Columbia University Medical Center between 11 AM and 1 PM. Time of day was kept consistent across assessments for each participant. Participants completed fullness ratings (visual analog scale, $0-100)$ after the ingestion of a small (250 kcal) fixed nutritionally complete liquid meal (Glytrol; Nestlé Nutrition, Vevey, Switzerland), used to minimize between subject differences in repletion. One hour after ingestion of the liquid meal, participants underwent an fMRI scan, during which they were presented with visual and auditory representations (cues) of high-ED foods (eg, pepperoni pizza, fudge sundae), low-ED foods (eg, raw vegetables) and neutral nonfoods (office supplies; eg, pencil, note pad). All high-ED stimuli had an ED at least $3.5 \mathrm{kcal} / \mathrm{g}$ and all low-ED stimuli had an ED less than $1 \mathrm{kcal} / \mathrm{g}^{26}$ (see Supplemental Table 1, Supplemental Digital Content available at: http://links.lww.com/SLA/A107). All procedures were identical pre- and postsurgery, and all surgical procedures were performed by the same surgeon.

\section{Stimuli Presentation Paradigm}

All cues were presented individually through 2 different modalities. Visual (pictorial) cues were transmitted through eye goggles and auditory (spoken name) cues were transmitted through a headset. The stimuli were presented in runs of 10 consecutive 4-second epochs (total block duration 40 seconds), with a 52 -second prerun baseline epoch and a 40 -second postrun baseline epoch. A Latin-Square paradigm employed 2 similar, but not identical, nonconsecutive runs of each type of food cue (high-ED, low-ED) for each condition (visual and auditory). The auditory stimuli were recorded 2-word names similar in content to the visual stimuli (eg, "chocolate brownie"), repeated twice to fill the 4-second epoch. Only areas activated in both stimuli presentation modalities were considered significant in image analyses. Fullness ratings were collected after meal ingestion, just before entering the fMRI scanner.

\section{fMRI Acquisition and Analyses}

A 1.5-T twin-speed fMRI scanner (GE Healthcare Technologies, Waukesha, Wisconsin) with quadrature RF head coil and $65-\mathrm{cm}$ bore diameter was used. Participants were positioned in the scanner with the head in a passive restraint. Three-plane localization was used to verify head position, and motion was minimized with restraint pads around the head and a tape strapped across the forehead. Total time in the scanner was about 60 minutes. In each run, 36 axial scans of the whole brain were acquired, each scan consisting of 25 
contiguous slices ( $4 \mathrm{~mm}$ thick), with a $19 \times 19 \mathrm{~cm}$ field of view, an acquisition matrix size of $128 \times 128$, and $1.5 \mathrm{~mm} \times 1.5 \mathrm{~mm}$ in plane resolution. The first 3 scans of each run (12 seconds) were discarded to attain magnetic equilibration. The axial slices were parallel to the AC/PC line. $\mathrm{T}^{*}$-weighted images with a gradient echo pulse sequence (echo time $=60$ $\mathrm{ms}$, repetition time $=4$ seconds, flip angle $=60$ degree angle) were acquired with matched anatomic high resolution $\mathrm{T} 1{ }^{*}$-weighted scans.

Functional data were analyzed with SPM5 (Wellcome Department of Imaging Neuroscience, London, United Kingdom). Before statistical analyses, the realigned T2* weighted volumes were slice-time corrected, spatially transformed to a standardized brain (Montreal Neurologic Institute) and smoothed with an $8 \mathrm{~mm}$ full-width half-maximum Gaussian kernel. First-level regressors were created by convolving the onset of each trial (audio and visual high-ED. low-ED, and nonfood) with the canonical HRF with duration of 40 seconds for both pre- and postsurgery scan sessions. Both scan sessions were included in the first level model for each participant. Average beta estimates of each condition (12 total per participant) were passed to a single second level model from which all subsequent contrasts of interested were generated. This model included an individual subject factor, and changes in BMI and fullness as additional nuisance regressors, to control for postsurgical weight loss (see Supplemental Table 2, Supplemental Digital Content, available at: http://links.lww.com/SLA/A107) and increases in postprandial fullness. Brain activation in response to visual and auditory representations of high-ED relative to low-ED foods was then conjoined to obtain areas of activation common across both stimuli presentation modalities.

Statistical maps for conjoint activation (eg, pre > post, highED-low-ED) were displayed at $P$ $<0.05$ uncorrected testing against the conjunction null hypothesis for effects of both visual and auditory modalities (Figs. 1 and 2)] and thresholded at cluster-size more than 20 (Table 1). To correct for multiple comparisons resulting from independent tests across many voxels, each region in Table 1 was checked for whether it appeared in a separate analysis. For this, statistical maps were reproduced using a threshold of $P<0.005$ uncorrected testing against the global null hypothesis (of 1 or more effects) and combined with a cluster-size threshold of 135 contiguous voxels, resulting in a threshold of $P<0.05$ corrected for multiple comparisons. The cluster threshold was determined by 1000 Monte Carlo simulations of whole-brain fMRI data with respective data parameters of the present study using the AlphaSim program as implemented in AFNI (version 2009). ${ }^{27}$ An uncorrected $P$ value of 0.005 under the global null hypothesis was applied; although not as conservative as the conjunction null, ${ }^{28}$ it was necessary in order to enable the application of cluster-based thresholding using the AlphaSim program. Regions that survived this threshold are denoted in Table 1 with an asterisk.

\section{Desire to Eat Ratings}

After each stimuli presentation run, participants were asked; "On a scale from 0 to 100, how much did what you just saw/heard make you want to eat, zero being "not at all" and 100 being "very much?" This question was not anchored to any particular cue; thus, desire to eat ratings were designed to reflect the extent to which exposure to food cues (high- vs. lowED) moderated each participant's desire to consume food, irrespective of what specific food(s) they desired. Ratings were averaged across all 4 runs from each food stimuli condition to obtain an overall desire to eat rating for each stimuli type for each participant, pre- and postsurgery. Individual pre- and postsurgical comparisons of desire to eat after exposure to high-ED versus low-ED food stimuli were calculated using independent samples $t$ tests. Changes in relative (high-ED-low-ED) desire to eat from pre- to postsurgery were calculated using repeated-measure analysis of variance. All non-fMRI statistical analyses were performed with SPSS v.16 (Chicago, Ill). 


\section{RESULTS}

\section{Changes in Brain Activation from Pre- to 1-Month Postsurgery}

Greater conjoint (visual + auditory) whole-brain activation, and conjoint activation in corticolimbic areas within the mesolimbic pathway, was found pre- as compared to postRYGB surgery in response to both high- and low-ED foods (Fig. 1). From pre- to postsurgery, greater reductions in activation were found in response to both high-ED and low-ED food stimuli relative to neutral non-food stimuli; however, postsurgical reductions in mesolimbic activity were found only in response to high-ED foods relative to nonfood stimuli (see Supplemental Figs. 1 and 2, Supplemental Digital Contents available at: http://links.lww.com/SLA/A108; http://links.lww.com/SLA/A109). The largest postsurgical reductions in activation were found in response to high-ED foods, and were most pronounced within the lingual gyrus, middle temporal gyrus, superior temporal gyrus, inferior parietal lobule and precuneus (see Supplemental Table 3, Supplemental Digital Content available at: http://links.lww.com/SLA/A107). Reductions in activation in response to low-ED foods were noted in many of the same areas but were smaller in magnitude (Fig. $1)$.

\section{Changes in Brain Activation in Response to High-relative to Low-ED Foods}

The pre- to postsurgical reduction in conjoint (visual + auditory) whole-brain activation in response to high-ED foods was more pronounced than the reduction in activation in response to lowED foods, particularly in corticolimbic areas within the mesolimbic pathway, including the VTA, ventral striatum, putamen, posterior cingulate and dmPFC (Fig. 1). Presurgery, the areas in which activation in response to high-ED foods was greater than activation in response to low-ED foods (high-ED-low-ED contrast) included the cingulate gyrus, thalamus, lentiform nucleus and caudate, anterior cingulate, medial frontal gyrus, superior frontal gyrus, inferior frontal gyrus, and middle frontal gyrus. Postsurgically, no clusters for the high-ED to low-ED contrast reached the applied cluster threshold, reflecting little difference between activation in response to high-ED versus low-ED foods after surgery (see Supplemental Fig. 3 Supplemental Digital Content, available at: http://links.lww.com/SLA/A110). Indeed, the difference between mesolimbic pathway activation in response to high-ED foods and that in response to low-ED foods (high-EDlow-ED contrast) was significantly greater pre- as compared to postsurgery. This pre to postsurgical decrease in activation in response to high-ED relative to low-ED foods was most pronounced and significant in the dIPFC, precuneus, dorsal cingulate, lentiform nucleus, and ventral striatum (Table 1, asterisks), indicating a selective postsurgical reduction in activation within these areas (Fig. 2). See Supplemental Discussion of Relevant Brain Areas, Supplemental Digital Content available at: http://links.lww.com/SLA/A107.

\section{Changes in Desire to Eat in Response to High-relative to Low-ED Food Cues}

Presurgery, desire to eat after exposure to high-ED food cues $(26.6 \pm 24.1)$ was significantly greater than the desire to eat after exposure to low-ED food cues $(15.1 \pm 19.5), t(9)=3.7, P$ $=0.005$. Postsurgery, however, subsequent desire to eat did not differ between exposure to high-ED (5.2 \pm 7.2$)$ and low-ED $(4.7 \pm 6.7)$ food cues, $t(9)=0.38, P=0.71$. The pre to postsurgical reduction in desire to eat after exposure to high-ED food cues $(-21.4 \pm 25.7, P$ $=0.028$ ) was greater than the non-significant reduction in desire to eat after exposure to lowED food cues $(-10.4 \pm 20.1, P=0.14), F(1,9)=11.9, P=0.007$, indicating a selective postsurgical reduction in the desire to eat after exposure to high-ED (vs. low-ED) food cues (Fig. 3). 


\section{DISCUSSION}

Results demonstrate that neural responses to food cues differ significantly from pre- to postRYGB surgery. The largest reductions in postsurgical activation in response to food cues were seen in a range of corticolimbic areas within the mesolimbic reward pathway, building on existing evidence for altered striatal dopamine receptor availability post-RYGB. ${ }^{17}$ Notably, of all the corticolimbic areas implicated, the most pronounced reductions in postsurgical activation were within the PFC, the primary integration site of reward-related information processing in the brain. ${ }^{29,30}$ Consistent with the notion that the brain mediates all nonreflexive behavior, ${ }^{30}$ postsurgical reductions in activation within brain areas associated with perceived (food) reward suggest some level of neural mediation of the decreases in caloric intake seen after RYGB surgery. In addition, mesolimbic pathway activation was preferentially reduced in response to high-(relative to low-) ED foods after RYGB surgery, indicating that the perceived reward value of foods typically judged to be hedonically pleasing such as pizza and cakes ${ }^{31}$ was reduced to a greater extent than was the perceived reward value of less hedonically pleasing foods such as vegetables. ${ }^{31-33}$

From pre- to postsurgery, all patients reported a reduction in appetitive responsivity or the desire to eat after exposure to food cues. However, desire to eat was also reduced to a greater extent after exposure to high-ED food cues as compared to low-ED food cues. These findings are consistent with reports ${ }^{9-12}$ suggesting that the desire to eat and intake of highcalorie foods are reduced significantly more than are the desire to eat and intake of lowcalorie foods after gastric bypass surgery. Thus, RYGB surgery seems to not only reduce overall appetite, but also the presurgical preference for high- relative to low-ED foods. This effect seems to be unrelated to postingestive side effects after surgery ${ }^{34}$ and has been proposed as a potentially significant mechanism of post-RYGB weight loss. ${ }^{35}$ These findings suggest that reductions in caloric intake and body weight seen after RYGB surgery may result not only from a reduction in the overall quantity of ingested food, but also from a decrease in the relative consumption of high- versus low-ED foods. Such changes in relative intake have previously been shown to reduce overall caloric intake and body weight. ${ }^{36,37}$

The selective reductions in both desire to eat and mesolimbic reward pathway activation in response to high- relative to lowED food cues provide additional evidence that the restrictive and malabsorptive mechanisms in RYGB do not fully account for post-surgical changes in appetite. $^{4-7,9-14}$ Mechanisms for these changes in desire to eat and neural activation, however, are yet to be delineated. It is possible that surgery leads to changes in operant conditioning (eg, high-calorie foods may be perceived of as less rewarding because of associations with the need for surgery or a less-positive presurgical self-image), which may be reflected by postsurgical reductions in mesolimbic activation in response to food cues. In addition, changes in gut peptide signaling may directly and indirectly influence (food) reward-related neural activation. For example, locally administered ghrelin has been shown to activate dopamine neurons in the VTA and promote dopamine turnover in the nucleus accumbens of the ventral striatum, and blocking ghrelin receptors in the VTA decreases food intake. ${ }^{38}$ Thus, postsurgical decreases in ghrelin levels ${ }^{39}$ may contribute to postsurgical decreases in mesolimbic activation and the perceived reward value of food. There is also evidence to suggest that postsurgical decreases in leptin ${ }^{40}$ may also reduce overall dopamine levels in the VTA and nucleus accumbens, ${ }^{41}$ potentially reducing the perceived reward value of food. ${ }^{42-44}$

Results in this study after surgical weight loss (11.8\% initial weight) in obese participants (mean initial body weight $=120.9 \mathrm{~kg}$ ) differ substantially from those found in the only known study of neural changes after nonsurgical weight loss (10\% initial) in obese participants (mean initial body weight $=118.2 \mathrm{~kg}$ ) ${ }^{21}$ where significant reductions in reward- 
related activation were not reported. Further, the postsurgical reduction in desire to eat highly palatable food in our study runs counter to that reported after nonsurgical weight loss. ${ }^{21,22}$ These results suggest that changes in neural and cognitive reactions to food cues may differ significantly after surgical versus nonsurgical weight loss, and that psychological motivation itself is unlikely to account for these changes. Further research will be necessary before causal inferences can be made.

The results of this study suggest that RYGB surgery may lead to a preferential reduction in the perceived reward value of, and desire to eat following exposure to, highly palatable high-ED food cues encountered in the external environment; both of which have been shown to predict food intake and body mass index. ${ }^{18,12,24,45}$ These data are consistent with current literature suggesting that the reductions in caloric intake seen after RYGB are at least partially mediated by neural signaling. ${ }^{4,5,7,13,14}$ In further identifying neural changes that result from RYGB surgery, it may become possible to mimic these effects pharmacologically, ${ }^{5,17}$ potentially providing a viable treatment option for the large proportion of clinically severely obese individuals who do not have access to bariatric surgery. ${ }^{46}$

\section{Limitations}

Although the sample size of 10 is relatively small, the longitudinal within subjects design provided sufficient power to detect effects in the primary outcomes, with the application of a k-correction threshold. ${ }^{27}$ and conjunction analyses. ${ }^{28}$ All participants were women, which increased statistical power by limiting within-sample variation, but also limits the generalizability of findings. Without a comparison group, alternative mediating factors cannot be ruled out; however, the temporal stability (retest reliability) of neural activation in fMRI ${ }^{4-50}$ suggests that the postsurgical changes in neural activity were due to the surgical procedure. Finally, postsurgical changes in desire to eat were not related to postsurgical weight loss at 1 month postsurgery. However, patients were on an all-liquid diet for the first postsurgical month, limiting the effect of food preferences on body weight during this period. Subsequent studies may assess food intake at extended postsurgical intervals (eg, 912 month) to determine whether postsurgical changes in brain activation predict changes in eating behavior and body weight.

\section{Supplementary Material}

Refer to Web version on PubMed Central for supplementary material.

\section{Acknowledgments}

This research was funded by the Columbia University Clinical and Translational Science Award Brain Imaging Core and the National Institutes of Health/the National Institute of Diabetes and Digestive and Kidney Diseases (R56 DK080153; R01DK080153). We thank Stephen Dashnaw and Andrew Kogan for conducting the fMRI scans, and Carl Dambkowski and Ku-Yu (Virginia) Chang for their assistance with manuscript preparation.

\section{References}

1. Ogden CL, Carroll MD, Curtin LR, et al. Prevalence of overweight and obesity in the United States, 1999-2004. JAMA. 2006; 295(13):1549-1555. [PubMed: 16595758]

2. Buchwald H, Avidor Y, Braunwald E, et al. Bariatric surgery: a systematic review and metaanalysis. JAMA. 2004; 292:1724-1737. [PubMed: 15479938]

3. Poves I, Cabrera M, Maristany C, et al. Gastrointestinal quality of life after laparoscopic Roux-en-Y gastric bypass. Obes Surg. 2006; 16:19-23. [PubMed: 16417752] 
4. Vincent RP, le Roux CW. Changes in gut hormones after bariatric surgery. Clin Endocrinol. 2008; 69(2):173-179.

5. Tadross JA, le Roux CW. The mechanisms of weight loss after bariatric surgery. Int J Obes. 2009; 33:S28-S32.

6. Brolin RE, LaMarca LB, Kenler HA, et al. Malabsorptive gastric bypass in patients with superobesity. J Gastrointest Surg. 2002; 6:195-205. [PubMed: 11992805]

7. Orlando FA, Goncalves CG, George ZM, et al. Neurohormonal pathways regulating food intake and changes after Roux-en-Y gastric bypass. Surg Obes Relat Dis. 2005; 1:486-495. [PubMed: 16925275]

8. Howard L, Malone M, Michalek A, et al. Gastric bypass and vertical banded gastroplasty--a prospective randomized comparison and 5-year follow-up. Obes Surg. 1995; 5:55-60. [PubMed: 10733794]

9. Thirlby R, Bahiraei F, Randall J, et al. Effect of Roux-en-y gastric bypass on satiety and food likes: the role of genetics. J Gastrointest Surg. 2006; 10:270-277. [PubMed: 16455461]

10. Tichansky DS, Boughter JD, Madan AK. Taste change after laparoscopic Roux-en-Y gastric bypass and laparoscopic adjustable gastric banding. Surg Obes Relat Dis. 2006; 2(4):440-444. [PubMed: 16925376]

11. Burge JC, Schaumburg JZ, Choban PS, et al. Changes in patients' taste acuity after Roux-en-Y gastric bypass for clinically severe obesity. J Am Diet Assoc. 1995; 95(6):666-670. [PubMed: 7759742]

12. Halmi KA, Mason E, Falk JR, et al. Appetitive behavior after gastric bypass for morbid obesity. Int J Obes. 1981; 5:457-464. [PubMed: 7309330]

13. Cummings DE, Overduin J, Foster-Schubert KE. Gastric bypass for obesity: mechanisms of weight loss and diabetes resolution. J Endocrin Metab. 2004; 89:2608-2615.

14. Cummings DE, Shannon MH. Roles for ghrelin in the regulation of appetite and body weight. Arch Surg. 2003; 138:389-396. [PubMed: 12686525]

15. Steele KE, Prokopowicz GP, Schweitzer MA, et al. Alterations of central dopamine receptors before and after gastric bypass surgery. Obes Surg. 2010; 20(3):369-374. [PubMed: 19902317]

16. Dunn JP, Cowan RL, Volkow ND, et al. Decreased dopamine type 2 receptor availability after bariatric surgery: preliminary findings. Brain Res. 2010; 1350:123-130. [PubMed: 20362560]

17. Berthoud H, Morrison C. The brain, appetite, and obesity. Annu Rev Psychol. 2008; 59:55-92. [PubMed: 18154499]

18. Lowe MR, van Steenburgh J, Ochner CN, et al. Individual differences in brain activation in relation to ingestive behavior and obesity. Physiol Behav. 2009; 5:561-571. [PubMed: 19361535]

19. Berthoud HR. Mind versus metabolism in the control of food intake and energy balance. Physiol Behav. 2004; 81:781-793. [PubMed: 15234184]

20. Lenard NR, Berthoud HR. Central and peripheral regulation of food intake and physical activity: pathways and genes. Obesity. 2008; 16:S11-S22. [PubMed: 19190620]

21. Rosenbaum M, Sy M, Pavlovich K, et al. Leptin reverses weight loss-induced changes in regional neural activity responses to visual food stimuli. J Clin Invest. 2008; 118:2583-2591. [PubMed: 18568078]

22. Doucet E, Imbeault P, St-Pierre S, et al. Appetite after weight loss by energy restriction and a lowfat diet-exercise follow-up. Int J Obes. 2000; 24:906-914.

23. Herman CP, Polivy J. External cues in the control of food intake in humans: the sensory-normative distinction. Physiol Behav. 2008; 94:722-728. [PubMed: 18499202]

24. Lowe MR, Butryn ML, Didie ER, et al. The power of food scale: a new measure of the psychological influence of the food environment. Appetite. 2009; 53:114-118. [PubMed: 19500623]

25. National Institutes of Health. Gastrointestinal surgery for severe obesity consensus statement. NIH Consensus Deve Conf. 1991; 9(1):1-20.

26. Rolls, BJ.; Barnett, R. Feel full on fewer calories. New York, NY: HarperCollins Publishers; 2000. Volumetrics. 
27. Cox RW. AFNI: software for analysis and visualization of functional magnetic resonance neuroimages. Comput Biomed Res. 1996; 29:162-173. [PubMed: 8812068]

28. Friston KJ, Penny WD, Glaser DE. Conjunction revisited. NeuroImage. 2005; 25:661-667. [PubMed: 15808967]

29. Ochner CN, Green D, van Steenburgh J, et al. Asymmetric prefrontal cortex activation in relation to markers of overeating in obese humans. Appetite. 2009; 53:44-49. [PubMed: 19426775]

30. Grobstein, P. Variability in brain function and behavior. In: Ramachandran, VS., editor. The Encyclopedia of Human Behavior. New York, NY: Academic Press; 1994. p. 447-458.

31. Nasser JA, Evans SM, Geliebter A, et al. Use of an operant task to estimate food reinforcement in adult humans with and without BED. Obesity. 2008; 16:1816-1820. [PubMed: 18535551]

32. Martel P, Fantino M. Mesolimbic dopaminergic system activity as a function of food reward: a microdialysis study. Pharmacol Biochem Behav. 1996; 53:221-226. [PubMed: 8848454]

33. de Araujo IE, Oliveira-Maia AJ, Sotnikova TD, et al. Food reward in the absence of taste receptor signaling. Neuron. 2008; 57:930-941. [PubMed: 18367093]

34. Thomas JR, Marcus E. High and low fat food selection with reported frequency intolerance following Roux-en Y gastric bypass. Obes Surg. 2008; 18:282-287. [PubMed: 18214629]

35. Miras A, le Roux CW. Bariatric surgery and taste: novel mechanisms of weight loss. Curr Opin Gastroen. 2010; 26:140-145.

36. Rolls BJ. The relationship between dietary energy density and energy intake. Physiol Behav. 2009; 97:609-615. [PubMed: 19303887]

37. Yao M, Roberts SB. Dietary energy density and weight regulation. Nutr Rev. 2001; 59:247-258. [PubMed: 11518179]

38. Jerlhag E, Egecioglu E, Dickson SL, et al. Ghrelin administration into tegmental areas stimulates locomotor activity and increases extracellular concentration of dopamine in the nucleus accumbens. Addict Biol. 2007; 12:6-16. [PubMed: 17407492]

39. Petereli R, Wolnerhanssen B, Peters T, et al. Improvement in glucose metabolism after bariatric surgery: comparison of laparoscopic Roux-en-Y gastric bypass and laparoscopic sleeve gastrectomy: a prospective randomized trial. Ann Surg. 2009; 250:234-241. [PubMed: 19638921]

40. Korner J, Inabnet W, Febres G, et al. Prospective study of gut hormone and metabolic changes after adjustable gastric banding and Roux-en-Y gastric bypass. Int J Obes (London). 2009; 33:786-795. [PubMed: 19417773]

41. Opland DM, Leinninger GM, Myers MG. Modulation of the mesolimbic dopamine system by leptin. Brain Res. 2010; 1350:65-70. [PubMed: 20417193]

42. Ochner CN, Gibson C, Carnell S, et al. Alterations in the neurohormonal regulation of energy intake through bariatric surgery for obesity. Physiol Behav. 2010; 100:549-559. [PubMed: 20452367]

43. Gibson C, Carnell S, Ochner CN, et al. Neuroimaging, gut peptides and obesity: novel studies of the neurobiology of appetite. J Neuroendocrinol. 2010; 22:833-845. [PubMed: 20553371]

44. Ochner, CN.; Gibson, C.; Shanik, M., et al. Changes in neurohormonal gut peptides following bariatric surgery. Int J Obes. 2010 July 13. [Epub ahead of print.10.1038/ijo.2010.132.]. Available at: http://www.nature.com/ijo/journal/vaop/ncurrent/abs/ijo2010132a.html

45. Forman EM, Hoffman KL, McGrath KB, et al. A comparison of acceptance-and control-based strategies for coping with food cravings: an analog study. Beh Res Therapy. 2007; 45:2372-2386.

46. Martin M, Beekley A, Kjorstad R, et al. Socioeconomic disparities in eligibility and access to bariatric surgery: a national population-based analysis. Surg Obes Relat Dis. 2010; 6:8-15. [PubMed: 19782647]

47. Manuck SB, Brown SM, Forbes EE, et al. Temporal stability of individual differences in amygdala reactivity. Am J Psychiatry. 2007; 164:1613-1614. [PubMed: 17898358]

48. Caceres A, Hall DL, Zelaya FO, et al. Measuring fMRI reliability with the intra-class correlation coefficient. Neuroimage. 2009; 45(3):758-768. [PubMed: 19166942]

49. Friedman L, Stern H, Brown GG, et al. Test-retest and between-site reliability in a multicenter fMRI study. Hum Brain Map. 2008; 29(8):958-972. 
50. Aron AR, Gluck MA, Poldrack RA. Long-term test-retest reliability of functional MRI in a classification learning task. Neuroimage. 2006; 29(3):1000-1006. [PubMed: 16139527] 
Pre $>1$-month Post

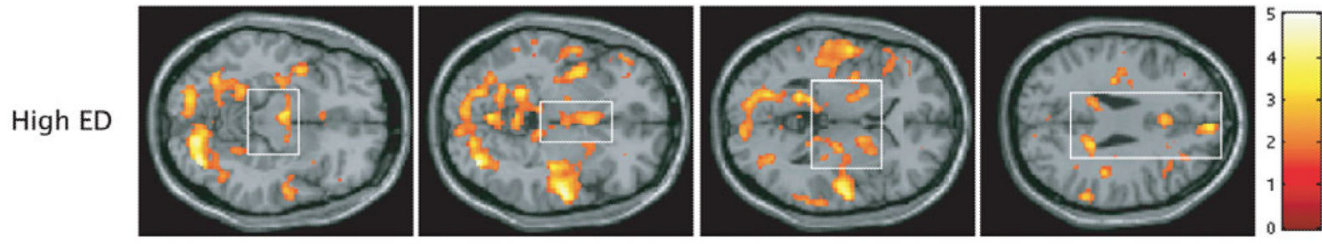

Low ED

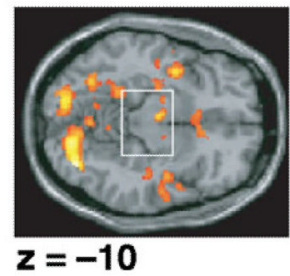

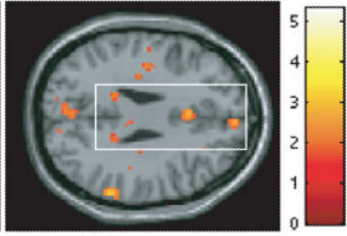

$z=28$

FIGURE 1.

Axial slices depicting areas in which activation was greater presurgery as compared to postsurgery across stimuli conditions. Relative to low-ED foods (bottom row), larger postsurgical reductions in conjoint (visual and auditory) activation (pre > postsurgery) in response to high-ED foods (top row) are seen in VTA $(z=-10)$, ventral striatum $(z=-4)$, putamen and lentiform nucleus $(z=2)$ posterior cingulate $(z=28$, left clusters $)$ and dmPFC $(z=28$, rightmost cluster). Montreal Neurological Institute coordinates for the axial slices are given below the statistical maps. The color bar represents $t$ values. For display purposes, activation maps are shown without a cluster extent threshold. 


\section{High-ED > Low-ED (Pre > 1-month Post)}
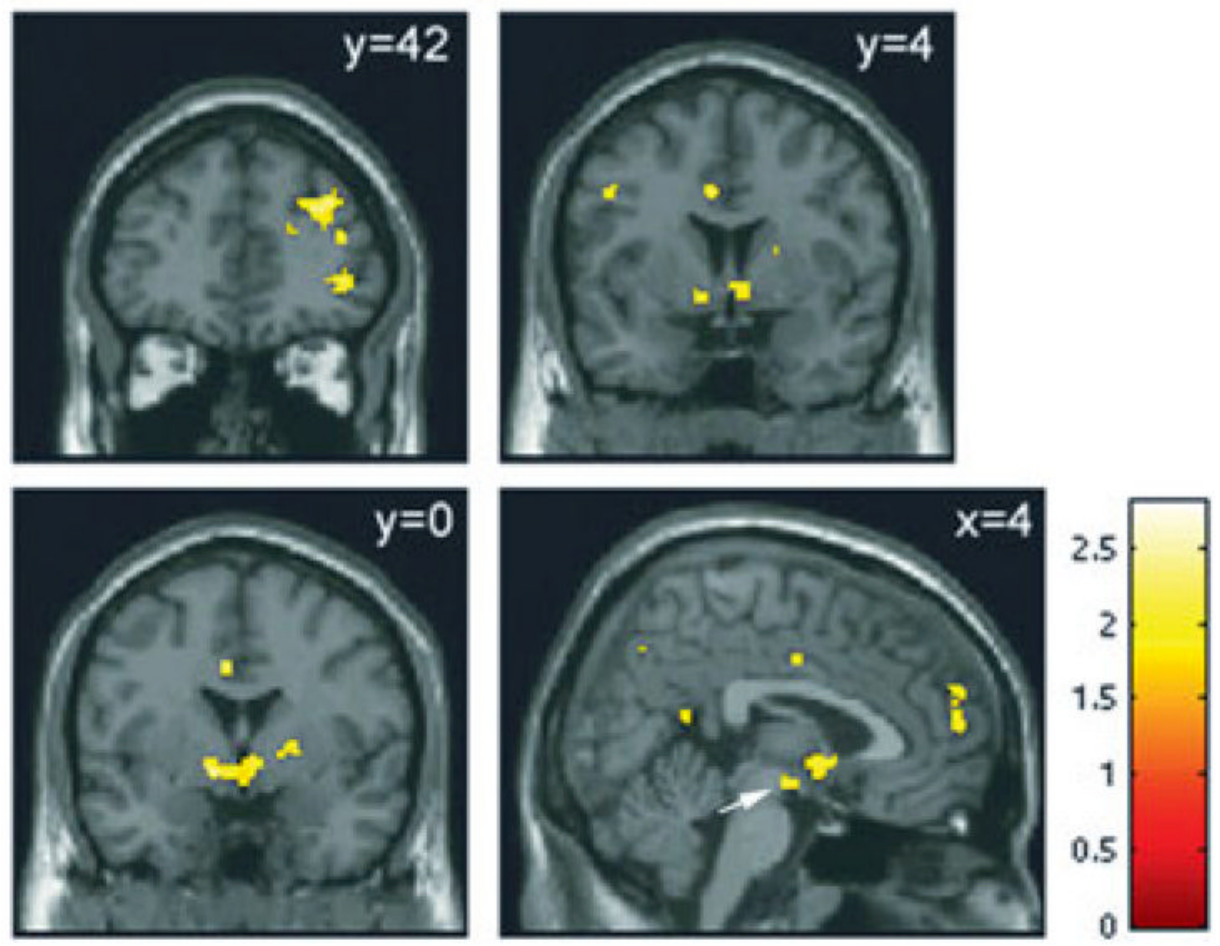

FIGURE 2.

Coronal and sagittal slices depicting areas in which the difference between conjoint activation in response to high- and low-ED foods (high-ED-low-ED contrast) was greater presurgery as compared to postsurgery. Montreal Neurological Institute coordinates are given in upper right corner of each panel. The color bar represents $t$ values. The largest clusters $(k>80)$ were seen in the dIPFC $(y=42$; topmost cluster), ventrolateral PFC (vlPFC; $y=42$; bottom cluster), ventral striatum ( $y=4$; bottom 2 clusters), putamen and lentiform nucleus ( $y=0$; bottom cluster), and $\operatorname{dmPFC}(x=4$; rightmost cluster). A small cluster ( $\mathrm{k}=$ 20) was also observed in the VTA $(x=4$; white arrow). Peak MNI coordinates for the above regions are listed in Table 1. 


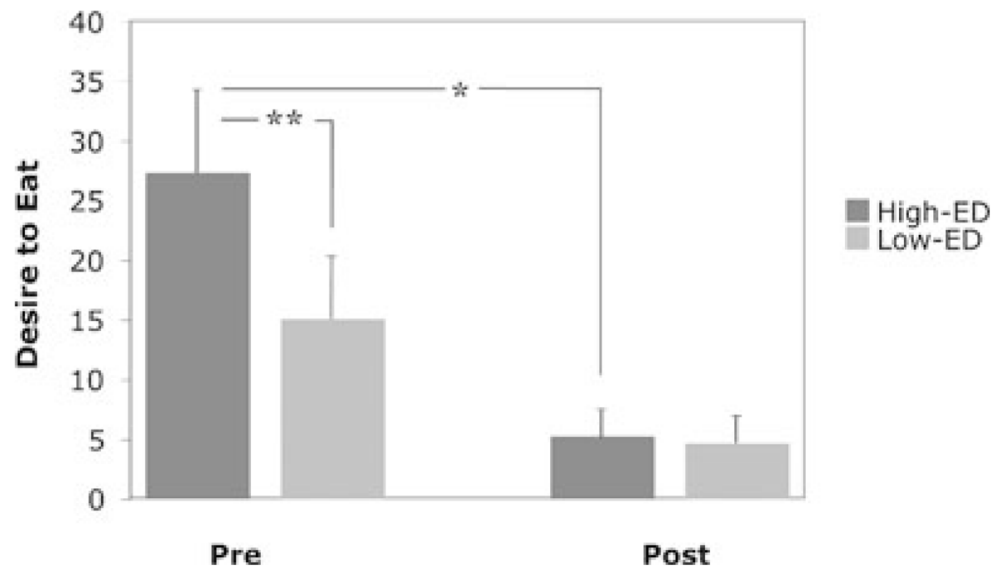

FIGURE 3.

The difference between the desire to eat following exposure to high-ED relative to low-ED foods (high-ED-low-ED) presurgery $(11.5 \pm 10)$ was greater than the non-significant highED to low-ED difference postsurgery $(0.5 \pm 4.2), F(1,9)=11.9, P=0.007 .{ }^{*} P<0.05 .{ }^{* *} P<$ 0.01 . 
TABLE 1

High-Low-ED Contrast, Pre > Post. ${ }^{\dagger}$

\begin{tabular}{lccc}
\hline Coordinates $(\boldsymbol{x}, \boldsymbol{y}, \boldsymbol{z})$ & Area(s) & $\mathbf{k}$ & Minimum $\boldsymbol{t}$ value \\
\hline $32,36,6$ & Inferior frontal gyrus (dlPFC) $^{*}$ & $253(333)$ & 2.59 \\
$-20,-64,30$ & Precuneus $^{*}$ & $234(144)$ & 2.81 \\
$30,42,30$ & dlPFC $^{*}$ & $121(333)$ & 2.25 \\
$28,22,32$ & & 47 & 2.32 \\
$-6,2,36$ & Dorsal cingulate $^{*}$ & $115(179)$ & 2.51 \\
$-12,2,-6$ & Lentiform nucleus $^{*}$ & $90(137)$ & 2.30 \\
$8,2,-4$ & Ventral striatum $^{*}$ & & 2.17 \\
$8,58,16$ & dmPFC $^{*}$ & 83 & 2.47 \\
$-36,-46,42$ & Inferior parietal lobe $^{*}$ & 80 & 2.28 \\
$50,-38,52$ & & 36 & 2.17 \\
$-62,-12,38$ & Precentral gyrus & 43 & 2.31 \\
$-56,-2,14$ & & 24 & 2.14 \\
$8,-52,14$ & Posterior cingulate & 42 & 2.07 \\
$-30,-58,60$ & Superior parietal lobe & 42 & 1.96 \\
$20,38,22$ & Anterior cingulate & 37 & 2.47 \\
$-8,32,30$ & & 28 & 2.09 \\
$-14,-22,4$ & Thalamus & 30 & 2.37 \\
$34,-70,6$ & Middle occipital gyrus & 26 & 2.36 \\
\hline
\end{tabular}

* Significant at $P<0.05$ corrected, when tested against the global null hypothesis (see the "Methods" section).

${ }^{\dagger}$ Areas showing a postsurgical reduction in the difference between conjoint activation in response to high-ED and low-ED foods. Italic values (in parentheses) indicate cluster size when threshold at $P<0.005$ was applied, testing against the global null hypothesis. Among these clusters, those larger than 135 voxels were deemed significant at $P<0.05$ corrected.

$\mathrm{k}$ indicates number of voxels within each cluster. 